\title{
Intuitionistic Fuzzy semiboundary and intuitionistic Fuzzy Product Related spaces
}

\author{
${ }^{1} \mathrm{~V}$. Thiripurasundari and ${ }^{2} \mathrm{~S}$. Murugesan \\ Department of Mathematics \\ ${ }^{1,2}$ Sri S.Ramasamy Naidu Memorial college, Sattur-626 203, India \\ E-mail: ${ }^{1}$ thiripurasund@gmail.com, ${ }^{2}$ satturmuruges@gmail.com
}

Keywords: Intuitionistic fuzzy topology, intuitionistic fuzzy topological spaces, intuitionistic fuzzy semi open set, intuitionistic fuzzy semi closed set, intuitionistic fuzzy semi boundary, intuitionistic fuzzy product related spaces, intuitionistic fuzzy semi continuous function.

\begin{abstract}
In this paper intuitionistic fuzzy semi boundary is introduced and its properties are investigated. Intuitionistic fuzzy semi continuous functions are characterized via intuitionistic fuzzy semi boundary.
\end{abstract}

\section{Introduction}

In the year 1965, Zadeh[10] introduced the concept of fuzzy sets. After that there have been a number of generalizations of this fundamental concepts. In the year 1986 Atanassov[1] introduced intuitionistic fuzzy set as a generalization of fuzzy set. Using the concept of intuitionistic fuzzy set, Coker[5] extended the concept of fuzzy topological spaces introduced by C.L.Chang[4] to intuitionistic fuzzy topological spaces. Pu and Liu[8] defined the notion of fuzzy boundary in fuzzy topological spaces in 1980. Properties of fuzzy boundary were investigated by Ahmed and Athar[2].

In this paper we introduce intuitionistic fuzzy semi boundary and investigate some of their properties. Further intuitionistic fuzzy semi boundary in product related spaces is analysed. Finally necessary conditions for intuitionistic fuzzy semi continuous functions are obtained via intuitionistic fuzzy semi boundary. Throughout this paper X,Y are non-empty sets.

\section{Preliminaries}

Definition 2.1: [1] An intuitionistic fuzzy set (IFS) $A$ in $X$ is an object having the form $\mathrm{A}=\left\{\left\langle x, \mu_{\mathrm{A}}(x), \gamma_{\mathrm{A}}(x)\right\rangle \mid x \in \mathrm{X}\right\}$ where the functions $\mu_{\mathrm{A}}: \mathrm{X} \rightarrow[0,1]$ and $\gamma_{\mathrm{A}}: \mathrm{X} \rightarrow[0,1]$ denote the degree of membership (namely, $\mu_{\mathrm{A}}(x)$ ) and the degree of non-membership (namely, $\gamma_{\mathrm{A}}(x)$ ) of each element $x \in X$ to the set A respectively, and

$0 \leq \mu_{\mathrm{A}}(x)+\gamma_{\mathrm{A}}(x) \leq 1$ for each $x \in X$

Definition 2.2: [1] Let A and B be IFS's of the forms $\mathrm{A}=\left\{\left\langle x, \mu_{\mathrm{A}}(x), \gamma_{\mathrm{A}}(x)\right\rangle \mid x \in \mathrm{X}\right\}$ and $\mathrm{B}=\left\{\left\langle x, \mu_{\mathrm{B}}(x), \gamma_{\mathrm{B}}(x)\right\rangle \mid x \in \mathrm{X}\right\}$. Then,

(a) $\mathrm{A} \subseteq \mathrm{B}$ if and only if $\mu_{\mathrm{A}}(x) \leq \mu_{\mathrm{B}}(x)$ and $\gamma_{\mathrm{A}}(x) \geq \gamma_{\mathrm{B}}(x)$ for all $x \in X$

(b) $\mathrm{A}=\mathrm{B}$ if and only if $\mathrm{A} \subseteq \mathrm{B}$ and $\mathrm{B} \subseteq \mathrm{A}$

(c) The complement of $\mathrm{A}$ is denoted by $\overline{\mathrm{A}}$ and is defined by $\bar{A}=\left\{\left\langle x, \gamma_{A}(x), \mu_{A}(x)\right\rangle \mid x \in X\right\}$,

(d) $A \cap B=\left\{\left\langle x, \mu_{A}(x) \wedge \mu_{B}(x), \gamma_{A}(x) \vee \gamma_{B}(x)\right\rangle \mid x \in X\right\}$,

(e) $\mathrm{A} \cup \mathrm{B}=\left\{\left\langle x, \mu_{\mathrm{A}}(x) \vee \mu_{\mathrm{B}}(x), \gamma_{\mathrm{A}}(x) \wedge \gamma_{\mathrm{B}}(x)\right\rangle \mid x \in \mathrm{X}\right\}$.

The IFS's $0_{\sim}=\{\langle x, \underline{0}, \underline{1}\rangle \mid x \in \mathrm{X}\}$ and $1_{\sim}=\{\langle x, \underline{1}, \underline{0}\rangle \mid x \in \mathrm{X}\}$ are respectively the empty set and the whole set of $\mathrm{X}$. For the sake of simplicity, we will use the notation $\mathrm{A}=\left\langle x, \mu_{\mathrm{A}}, \gamma_{\mathrm{A}}\right\rangle$ instead of $A=\left\{\left\langle x, \mu_{A}(x), \gamma_{A}(x)\right\rangle \mid x \in X\right\}$. 
Definition 2.3: [1] Let $X$ and $Y$ be IFTS's and $\mathrm{f}: \mathrm{X} \rightarrow \mathrm{Y}$ be a function. If $\mathrm{B}=\left\{\left\langle y, \mu_{\mathrm{B}}(y), \gamma_{\mathrm{B}}(y)\right\rangle \mid y \in \mathrm{Y}\right\}$ is an IFS in $\mathrm{Y}$, then the pre image of $\mathrm{B}$ under, denoted by $\mathrm{f}^{-1}(\mathrm{~B})$ is the IFS in $\mathrm{X}$ defined by

$$
\mathrm{f}^{-1}(\mathrm{~B})=\left\{\left\langle x, \mathrm{f}^{-1}\left(\mu_{\mathrm{B}}\right)(x), \mathrm{f}^{-1}\left(\gamma_{\mathrm{B}}\right)(x)\right\rangle \mid x \in \mathrm{X}\right\}
$$

and the image of $A$ under, denoted by $\mathrm{f}(\mathrm{A})=\left\langle\mathrm{y}, \mathrm{f}\left(\mu_{\mathrm{A}}\right), \mathrm{f}\left(\gamma_{\mathrm{A}}\right)\right\rangle$ is an IFS of Y, where for each $y \in Y$

$$
\begin{gathered}
\mathrm{f}\left(\mu_{\mathrm{A}}\right)(y)=\left\{\begin{array}{cl}
\sup _{x \in \mathrm{f}^{-1}(y)} & \mu_{\mathrm{A}}(x) \\
0 & \text { if } \mathrm{f}^{-1}(y) \neq \emptyset
\end{array}\right. \\
\mathrm{f}\left(\gamma_{\mathrm{A}}\right)(y)=\left\{\begin{array}{cl}
\inf _{x \in \mathrm{f}^{-1}(y)} \gamma_{\mathrm{A}}(x) & \text { if } \mathrm{f}^{-1}(y) \neq \emptyset \\
1 & \text { otherwise. }
\end{array}\right.
\end{gathered}
$$

It is noted that $\overline{f^{-1}(B)}=f^{-1}(\bar{B})$

Definition 2.4: [5] An intuitionistic fuzzy topology (IFT) on $X$ is a family $\tau$ of IFS's in $X$ satisfying the following axioms:

(1) $0_{\sim}, 1_{\sim} \in \tau$,

(2) $G_{1} \cap G_{2} \in \tau$ for any $G_{1} G_{2} \in \tau$,

(3) $\cap \mathrm{G}_{\mathrm{i}} \in \tau$ for any family $\left\{\mathrm{G}_{\mathrm{i}} / \mathrm{i} \in \mathrm{J}\right\} \tau$.

In this case, the pair $(\mathrm{X}, \tau)$ is called an intuitionistic fuzzy topological space(IFTS) and any IFS in $\tau$ is known as an intuitionistic fuzzy open set (IFOS) in X. The complement $\bar{A}$ of an IFOS A in IFTS $(\mathrm{X} \tau)$ is called an intuitionistic fuzzy closed set (IFCS) in X.

Definition 2.5: [5] Let $(\mathrm{X}, \tau)$ be an IFTS and let $\mathrm{A}=\left\langle x, \mu_{\mathrm{A}}, \gamma_{\mathrm{A}}\right\rangle$ be an IFS in $\mathrm{X}$. Then the intuitionistic fuzzy interior and intuitionistic fuzzy closure of A are defined by

$$
\begin{aligned}
& \operatorname{Int}(A)=U\{G \mid G \text { is an IFOS in } X \text { and } G \subseteq A\}, \\
& C l(A)=\bigcap\{K \mid K \text { is an IFCS in } X \text { and } A \subseteq K\} .
\end{aligned}
$$

Properties of closure and interior of a intuitionistic fuzzy set which are needed in the sequel, are summarized in the following lemma.

Lemma 2.6: For any IFS's A and B in IFTS $(X, \tau)$,

(1) $\operatorname{Cl}(\overline{\mathrm{A}})=\overline{\operatorname{Int}(\mathrm{A})}$;

(2) $\operatorname{Int}(\overline{\mathrm{A}})=\overline{\mathrm{Cl}(\mathrm{A})}$;

(3) $\mathrm{A}$ is an intuitionistic fuzzy closed (IFC) $\Leftrightarrow \mathrm{Cl}(\mathrm{A})=\mathrm{A}$ (resp. intuitionistic fuzzy open (IFO) $\Leftrightarrow$ $\operatorname{Int}(\mathrm{A})=\mathrm{A})$

(4) $\mathrm{A} \leq \mathrm{B} \Longrightarrow C l(\mathrm{~A}) \leq C l(\mathrm{~B}) ;(\operatorname{Int}(\mathrm{A}) \leq \operatorname{Int}(\mathrm{B}))$;

(5) $\mathrm{ClCl}(\mathrm{A})=\mathrm{Cl}(\mathrm{A}) ;(\operatorname{Int} \operatorname{Int}(\mathrm{A})=\operatorname{Int}(\mathrm{A}))$;

(6) $\mathrm{Cl}(\mathrm{A}) \vee \mathrm{Cl}(\mathrm{B})=\mathrm{Cl}(\mathrm{A} \vee \mathrm{B})$;

(7) $\mathrm{Cl}(\mathrm{A}) \wedge \mathrm{Cl}(\mathrm{B}) \geq \mathrm{Cl}(\mathrm{A} \wedge \mathrm{B})$;

(8) $\operatorname{Int}(A) \vee \operatorname{Int}(B) \leq \operatorname{Int}(A \vee B)$;

(9) $\operatorname{Int}(A) \wedge \operatorname{Int}(B)=\operatorname{Int}(A \wedge B)$.

Definition 2.7: [9] Let A be a IFS in an IFTS (X, $\square$ ). Then the intuitionistic fuzzy boundary of A is defined as ${ }^{\mathrm{IBd} A}=\mathrm{Cl} \mathrm{A} \wedge \mathrm{Cl} \overline{\mathrm{A}}$. IBdAis a intuitionistic fuzzy closed $\operatorname{set}(\mathrm{IFCS})$. 


\section{Intuitionistic Fuzzy Semiboundary}

Definition 3.1: [6] An IFS A in an IFTS $(X, \tau)$ is called an intuitionistic fuzzy semi open set(IFSOS) if $A \subseteq c l$ (int(A)) (or) if there exists $\mathrm{V} \in \tau$ such that $V \leq A \leq C l V$.

An IFS A is called intuitionistic fuzzy semi-closed set, if the complement of is an IFSOS.

Definition 3.2: [6] Let A be a IFS in an IFTS $(X, \tau)$. Then Semi interior(SInt) and semi closure $(\mathrm{SCl})$ of $\mathrm{A}$ are given as

$$
\begin{aligned}
& \text { SInt } A=U\{G / G \text { is an IFSOS in } X, G \subseteq A\} \\
& S C l A=\cap\{K / K \text { is an IFSCS in } X, A \subseteq K\}
\end{aligned}
$$

Theorem 3.3: For IFSs A and $B$ in an IFTS $(X, \tau)$. Then

(1) $\operatorname{SInt}(A \vee B) \geq \operatorname{SInt}(A) \vee(\operatorname{SIntB})$;

(2) $\operatorname{SInt}(A \wedge B)=\operatorname{SInt}(A) \wedge(\operatorname{SInt} B)$;

(3) $\mathrm{SCl}(\mathrm{A} \vee \mathrm{B})=\mathrm{SCl}(\mathrm{A}) \vee \mathrm{SCl}(\mathrm{B})$

(4) $\mathrm{SCl}(\mathrm{A} \wedge \mathrm{B}) \leq \mathrm{SCl}(\mathrm{A}) \wedge \mathrm{SCl}(\mathrm{B})$;

(5) $\mathrm{SCl} \mathrm{SCl}(\mathrm{A})=\mathrm{SCl}(\mathrm{A})$

(6) $\operatorname{Sint} \operatorname{SInt}(A)=\operatorname{SInt}(A)$

(7) $\overline{\operatorname{SCl}(\mathrm{A})}=\operatorname{Sint}(\overline{\mathrm{A}})$;

(8) $\overline{\operatorname{SInt}(A)}=\operatorname{SCl}(\bar{A})$.

Proof: (1) SInt (A) and $\operatorname{SInt}(B)$ are both intuitionistic fuzzy semiopen and $A \leq A \vee B, B \leq A \vee B \Rightarrow$ $\operatorname{SInt}(A) \leq \operatorname{SInt}(A \vee B)$ and $\operatorname{SInt}(B) \leq \operatorname{SInt}(A \vee B)$. Hence, $\operatorname{SInt}(A) \vee \operatorname{SInt}(B) \leq \operatorname{SInt}(A \vee B)$.

(2) $\mathrm{A} \wedge \mathrm{B} \leq \mathrm{A}$ and $\mathrm{A} \wedge \mathrm{B} \leq \mathrm{B} \Rightarrow \operatorname{SInt}(\mathrm{A} \wedge \mathrm{B}) \leq \operatorname{SInt}(\mathrm{A}) \wedge \operatorname{SInt}(\mathrm{B})$.

$\operatorname{SInt}(A \wedge B) \leq \operatorname{SInt}(B)$. Therefore $\operatorname{SInt}(A \wedge B) \leq \operatorname{SInt}(A) \wedge \operatorname{SInt}(B)$.

Conversely, $\operatorname{SInt}(\mathrm{A}) \leq \mathrm{A}$ and $\operatorname{SInt}(\mathrm{B}) \leq \mathrm{B} \Rightarrow \operatorname{SInt}(\mathrm{A}) \wedge \operatorname{SInt}(\mathrm{B}) \leq \mathrm{A} \wedge \mathrm{B}$ and $\operatorname{SInt}(\mathrm{A}) \wedge \mathrm{SInt}(\mathrm{B})$ is intuitionistic fuzzy semiopen. But SInt $(A \wedge B) s$ the largest intuitionistic fuzzy semiopen set contained in $A \wedge B$. Therefore SInt $(A) \wedge \operatorname{SInt}(B) \leq \operatorname{SInt}(A \wedge B)$ Hence SInt $(A) \wedge \operatorname{SInt}(B)=\operatorname{SInt}$ $(\mathrm{A} \wedge \mathrm{B})$

(3) It follows easily from (2).

(4) Since $\mathrm{A} \wedge \mathrm{B} \leq \mathrm{A}, \mathrm{A} \wedge \mathrm{B} \leq \mathrm{B} \Rightarrow \mathrm{SCl}(\mathrm{A} \wedge \mathrm{B}) \leq \mathrm{SCl}(\mathrm{A})$,

$\mathrm{SCl}(\mathrm{A} \wedge \mathrm{B}) \leq \mathrm{SCl}(\mathrm{B})$ Hence, $\mathrm{SCl}(\mathrm{A} \wedge \mathrm{B}) \leq \mathrm{SCl}(\mathrm{A}) \wedge \mathrm{SCl}(\mathrm{B})$

(5) $-(8)$ proofs are straight forward.

In theorem3.3 ( 1 and 4$)$, the equality may not holds as seen in the following example.

Example 3.4: Let $X=\{a, b\}$ and let $A=\left\langle x, \frac{0.6}{\mathrm{a}}+\frac{0.5}{\mathrm{~b}}, \frac{0.4}{\mathrm{a}}+\frac{0.3}{\mathrm{~b}}\right\rangle$. Then

$\tau=\left\{0_{\sim}, 1_{\sim}, \mathrm{A}\right\}$ is an IFT on X. $\tau^{\prime}=\left\{0_{\sim}, 1_{\sim},\left\langle x, \frac{0.4}{\mathbf{a}}+\frac{0.3}{\mathrm{~b}}, \frac{0.6}{\mathrm{a}}+\frac{0.5}{\mathrm{~b}}\right\rangle\right\}$.

$\operatorname{IFSOS}(\mathrm{X})=\left\{0_{\sim}, 1_{\sim},\left\langle x, \frac{l_{1}}{a}+\frac{l_{2}}{b}, \frac{m_{1}}{a}+\frac{m_{2}}{b}\right\rangle\right.$ where $0.6 \leq l_{1} \leq 1,0.5 \leq l_{2} \leq 1$,

$0 \leq m_{1} \leq 0.4,0 \leq m_{2} \leq 0.3$.

$\operatorname{IFSCS}(\mathrm{X})=\left\{0_{\sim}, 1_{\sim},\left\langle x, \frac{\alpha_{1}}{a}+\frac{\alpha_{2}}{b}, \frac{\beta_{1}}{a}+\frac{\beta_{2}}{b}\right\rangle\right.$ where $0 \leq \alpha_{1} \leq 0.4,0 \leq \alpha_{2} \leq 0.3$.

$0.6 \leq \beta_{1} \leq 1,0.5 \leq \beta_{2} \leq 1$.

Let

$\mathrm{B}=\left\langle x, \frac{0.6}{\mathrm{a}}+\frac{0.2}{\mathrm{~b}}, \frac{0.4}{\mathrm{a}}+\frac{0.5}{\mathrm{~b}}\right\rangle$ and $\mathrm{C}=\left\langle x, \frac{0.3}{\mathrm{a}}+\frac{0.5}{\mathrm{~b}}, \frac{0.6}{\mathrm{a}}+\frac{0.3}{\mathrm{~b}}\right\rangle$. 
Then, $\mathrm{B} \wedge \mathrm{C}=\left\langle x, \frac{0.3}{\mathrm{a}}+\frac{0.2}{\mathrm{~b}}, \frac{0.6}{\mathrm{a}}+\frac{0.5}{\mathrm{~b}}\right\rangle, \mathrm{B} \vee \mathrm{C}=\left\langle x, \frac{0.6}{\mathrm{a}}+\frac{0.5}{\mathrm{~b}}, \frac{0.4}{\mathrm{a}}+\frac{0.3}{\mathrm{~b}}\right\rangle, \mathrm{SCl} \mathrm{B}=1_{\sim}$,

$\operatorname{SCl} \mathrm{C}=1_{\sim}, \operatorname{SInt} \mathrm{B}=0_{\sim}, \operatorname{SInt} \mathrm{C}=0_{\sim}, \operatorname{SCl}(\mathrm{B} \wedge \mathrm{C})=\left\langle x, \frac{0.3}{\mathbf{a}}+\frac{0.2}{\mathbf{b}}, \frac{0.6}{\mathbf{a}}+\frac{0.5}{\mathrm{~b}}\right\rangle$,

$\operatorname{SInt}(B \vee C)=\left\langle x, \frac{0.6}{\mathrm{a}}+\frac{0.5}{\mathrm{~b}}, \frac{0.4}{\mathrm{a}}+\frac{0.3}{\mathrm{~b}}\right\rangle$.

Hence,

$\operatorname{SInt}(B \vee C)=\left\langle x, \frac{0.6}{a}+\frac{0.5}{b}, \frac{0.4}{a}+\frac{0.3}{b}\right\rangle \neq 0 \sim=\operatorname{SInt}(B) \vee \operatorname{SInt}(C)$,

$\operatorname{SCl}(\mathrm{B} \wedge \mathrm{C})=\left\langle x, \frac{0.3}{\mathrm{a}}+\frac{0.2}{\mathrm{~b}}, \frac{0.6}{\mathrm{a}}+\frac{0.5}{\mathrm{~b}}\right\rangle \neq 1_{\sim}=\mathrm{SCl}(\mathrm{B}) \wedge \mathrm{SCl}(\mathrm{C})$.

Definition 3.5: Let A be a IFS in an IFTS $(X, \tau)$. Then the intuitionistic fuzzy semiboundary of $A$ is defined as ISBd $\mathrm{A}=\mathrm{SCl} \mathrm{A} \wedge \mathrm{SCl} \overline{\mathrm{A}}$. ISBd $\mathrm{A}$ is a intuitionistic fuzzy semiclosed set(IFSCS).

Remark 3.6: In classical topology, for an arbitrary set A of a topological space X, A U SBd A = SCl A, but $\mathrm{A} \vee \mathrm{ISBd} \mathrm{A} \leq \mathrm{SCl} \mathrm{A}$, for an arbitrary intuitionistic fuzzy set A in an IFTS $(\mathrm{X}, \tau)$, where the equality may not hold as is seen in the following example.

Example 3.7: Let $X=\{a, b\}$ and let $A=\left\langle x, \frac{0.2}{\mathrm{a}}+\frac{0.3}{\mathrm{~b}}, \frac{0.4}{\mathrm{a}}+\frac{0.6}{\mathrm{~b}}\right\rangle$ Then $\tau=\left\{0_{\sim}, 1_{\sim}, \mathrm{A}\right\}$ is an IFTS on $\mathrm{X}$, $\tau^{\prime}=\left\{0_{\sim}, 1 \sim,\left\langle x, \frac{0.4}{\mathrm{a}}+\frac{0.6}{\mathrm{~b}}, \frac{0.2}{\mathrm{a}}+\frac{0.3}{\mathrm{~b}}\right\rangle\right\}$. IFSOS$(\mathrm{X})=\left\{0_{\sim}, 1_{\sim},\left\langle x, \frac{l_{1}}{a}+\frac{l_{2}}{b}, \frac{m_{1}}{a}+\frac{m_{2}}{b}\right\rangle\right.$ where $0.2 \leq l_{1} \leq 0.4,0.3 \leq l_{2} \leq 0.6$, $0.2 \leq m_{1} \leq 0.4,0.3 \leq m_{2} \leq 0.6$

$\operatorname{IFSCS}(\mathrm{X})=\left\{0_{\sim}, 1_{\sim},\left\langle x, \frac{\alpha_{1}}{a}+\frac{\alpha_{2}}{b}, \frac{\beta_{1}}{a}+\frac{\beta_{2}}{b}\right\rangle\right.$ where $0.2 \leq \alpha_{1} \leq 0.4,0.3 \leq \alpha_{2} \leq 0.6$.

$0.2 \leq \beta_{1} \leq 0.4,0.3 \leq \beta_{2} \leq 0.6$.

Choose, $\mathrm{P}=\left\langle x, \frac{0.5}{\mathrm{a}}+\frac{0.6}{\mathrm{~b}}, \frac{0.4}{\mathrm{a}}+\frac{0.3}{\mathrm{~b}}\right\rangle$ be IFTS on $\mathrm{X}$.

Then $\mathrm{SCl} \mathrm{P}=1_{\sim}, \operatorname{SCl} \overline{\mathrm{P}}=\left\langle x, \frac{0.4}{\mathrm{a}}+\frac{0.3}{\mathrm{~b}}, \frac{0.4}{\mathrm{a}}+\frac{0.6}{\mathrm{~b}}\right\rangle$,

ISBd P $=S C l P \wedge S C l \bar{P}=\left\langle x, \frac{0.4}{\mathrm{a}}+\frac{0.3}{\mathrm{~b}}, \frac{0.4}{\mathrm{a}}+\frac{0.6}{\mathrm{~b}}\right\rangle$.

Hence, $\mathrm{P} \vee \operatorname{ISBd} \mathrm{P}=\left\langle x, \frac{0.5}{\mathrm{a}}+\frac{0.6}{\mathrm{~b}}, \frac{0.4}{\mathrm{a}}+\frac{0.3}{\mathrm{~b}}\right\rangle \neq 1=\mathrm{SCl} \mathrm{P}$,

Proposition 3.8: Let A and B be IFS's in an IFTS $(X, \tau)$. Then the following conditions hold.

(1) ISBd A= ISBd $\bar{A}$

(2) If A is IFSC, then ISBd A $\leq \mathrm{A}$

(3) If $\mathrm{A}$ is IFSO, then ISBd $\mathrm{A} \leq{ }^{-} \mathrm{A}$

(4) Let $\mathrm{A} \leq \mathrm{B}$ and $\mathrm{B} \in \operatorname{IFSCS}(\mathrm{X})($ resp., $\mathrm{B} \in \operatorname{IFSCS}(\mathrm{X}))$. Then, ISBd $\mathrm{A} \leq \mathrm{B}$

(resp., ISBd $\mathrm{A} \leq \overline{\mathrm{B}}$ );

(5) $(\overline{\operatorname{ISBd} A})=\operatorname{SInt} A \vee \operatorname{SInt} \bar{A}$;

(6) ISBd A $\leq$ IBd A;

(7) $\operatorname{SCl}(\operatorname{ISBd} A) \leq \operatorname{IBd} A$

Proof: (1) ISBd A $=\mathrm{SCl} A \wedge \mathrm{SCl} \overline{\mathrm{A}}=\mathrm{SCl} \overline{\mathrm{A}} \wedge \mathrm{SCl} \mathrm{A}$

$$
=\operatorname{SCl} \overline{\mathrm{A}} \wedge \mathrm{SCl} \overline{\overline{\mathrm{A}}}=\mathrm{ISBd} \overline{\mathrm{A}}
$$


(2) $\operatorname{ISBd} \mathrm{A}=\mathrm{SCl} \mathrm{A} \wedge \mathrm{SCl} \overline{\mathrm{A}} \leq \mathrm{SCl} \mathrm{A}=\mathrm{A}$ Hence ISBd A $\leq \mathrm{A}$.

(3) A is IFSO implies $\bar{A}$ IFSC. By (1), (2) $\operatorname{ISBd} \bar{A} \leq \bar{A}$ Hence ISBd $A \leq \bar{A}$.

(4) Since $\mathrm{A} \leq \mathrm{B}$ implies $\mathrm{SCl} \mathrm{A} \leq \mathrm{SCl} \mathrm{B}$

$\mathrm{ISBd} \mathrm{A}=\mathrm{SCl} \mathrm{A} \wedge \mathrm{SCl} \overline{\mathrm{A}} \leq \mathrm{SCl} \mathrm{B} \wedge \mathrm{SCl} \overline{\mathrm{A}} \leq \mathrm{SCl} \mathrm{B}=\mathrm{B}$. Hence, $\operatorname{ISBd} \mathrm{A} \leq \mathrm{B}$.

(5) $\overline{\mathrm{ISBdA}}=(\overline{\operatorname{SCl} \mathrm{A} \wedge \mathrm{SCl} \overline{\mathrm{A}}})=(\overline{\operatorname{SCl} \mathrm{A}}) \vee(\overline{\operatorname{SCl} \overline{\mathrm{A}}})=\operatorname{Sint} \overline{\mathrm{A}} \vee \operatorname{Sint} \overline{\overline{\mathrm{A}}}$

$$
=\operatorname{SInt} \overline{\mathrm{A}} \vee \operatorname{SInt} \mathrm{A} \text {. Hence, } \overline{\mathrm{ISBd} A}=\operatorname{SInt} \mathrm{A} \vee \operatorname{SInt} \overline{\mathrm{A}} \text {. }
$$

(6) Since $\mathrm{SCl} \mathrm{A} \leq \mathrm{Cl} \mathrm{A}$ and $\mathrm{SCl} \overline{\mathrm{A}} \leq \mathrm{Cl} \overline{\mathrm{A}}$.

Then ISBd A $=\mathrm{SCl} A \wedge \mathrm{SCl} \overline{\mathrm{A}} \leq \mathrm{Cl} A \wedge \mathrm{Cl} \overline{\mathrm{A}} \leq \mathrm{IBd}$ A. Hence, ISBd A $\leq \mathrm{IBd} \mathrm{A}$.

(7) $\operatorname{SCl}(\operatorname{ISBd} \mathrm{A})=\operatorname{SCl}(\mathrm{SCl} A \wedge \mathrm{SCl} \overline{\mathrm{A}}) \leq \mathrm{SCl} \operatorname{SCl} \mathrm{A} \wedge \mathrm{SCl} \operatorname{SCl} \overline{\mathrm{A}}=\operatorname{SCl} \mathrm{A} \wedge \mathrm{SCl} \overline{\mathrm{A}}$

$=$ ISBd A $\leq$ IBd Hence, $S C l(\operatorname{ISBd} A) \leq \operatorname{IBd~A}$.

The converse of (2), (3) and the equality may hold in (6), (7) of Proposition 3.8 is not true as seen in the following example.

Example 3.9: Choose IFSs $P=\left\langle x, \frac{0.5}{a}+\frac{0.6}{b}, \frac{0.4}{a}+\frac{0.3}{b}\right\rangle$

$\mathrm{Q}=\left\langle x, \frac{0.3}{\mathrm{a}}+\frac{0.4}{\mathrm{~b}}, \frac{0.6}{\mathrm{a}}+\frac{0.4}{\mathrm{~b}}\right\rangle$ in the IFTS $(\mathrm{X} \tau)$ defined in example 3.7.

Then(1) $\operatorname{SCl} \mathrm{P}=1 \sim, \operatorname{SCl} \overline{\mathrm{P}}=\left\langle x, \frac{0.4}{\mathrm{a}}+\frac{0.3}{\mathrm{~b}}, \frac{0.4}{\mathrm{a}}+\frac{0.6}{\mathrm{~b}}\right\rangle$,

ISBd $\mathrm{P}=\left\langle x, \frac{0.4}{\mathrm{a}}+\frac{0.3}{\mathrm{~b}}, \frac{0.4}{\mathrm{a}}+\frac{0.6}{\mathrm{~b}}\right\rangle \leq \mathrm{P}$. but $\mathrm{P}$ is not intuitionistic fuzzy semi closed.

(2) $\mathrm{SCl} \mathrm{Q}=\left\langle x, \frac{0.3}{\mathrm{a}}+\frac{0.4}{\mathrm{~b}}, \frac{0.4}{\mathrm{a}}+\frac{0.4}{\mathrm{~b}}\right\rangle, \operatorname{SCl} \overline{\mathrm{Q}}=1_{\sim}$,

ISBd $Q=\left\langle x, \frac{0.3}{\mathrm{a}}+\frac{0.4}{\mathrm{~b}}, \frac{0.4}{\mathrm{a}}+\frac{0.4}{\mathrm{~b}}\right\rangle \leq \overline{\mathrm{Q}}$.

but $\mathrm{Q}$ is not intuitionistic fuzzy semi open.

(3)

$\mathrm{Cl} \mathrm{P}=1_{\sim}, \mathrm{Cl} \overline{\mathrm{P}}=\left\langle x, \frac{0.4}{\mathrm{a}}+\frac{0.6}{\mathrm{~b}}, \frac{0.2}{\mathrm{a}}+\frac{0.3}{\mathrm{~b}}\right\rangle, \mathrm{IBd} \mathrm{P}=\left\langle x, \frac{0.4}{\mathrm{a}}+\frac{0.6}{\mathrm{~b}}, \frac{0.2}{\mathrm{a}}+\frac{0.3}{\mathrm{~b}}\right\rangle$.

Hence, ISBd P $\lesseqgtr$ IBd P

(4) $\operatorname{SCl}(\operatorname{ISBd} \mathrm{P})=\left\langle x, \frac{0.4}{\mathrm{a}}+\frac{0.3}{\mathrm{~b}}, \frac{0.4}{\mathrm{a}}+\frac{0.6}{\mathrm{~b}}\right\rangle \varsubsetneqq \mathrm{IBd} \mathrm{P}$.

Proposition 3.10: Let A be a intuitionistic fuzzy set in an IFTS $(X \tau)$. Then,

(1) $\operatorname{ISBd} A=\operatorname{SCl} A \wedge(\overline{\operatorname{SInt} A})$;

(2) $\operatorname{ISBd}(\operatorname{SInt} A) \leq \operatorname{ISBd} A$;

(3) $\operatorname{ISBd}(\mathrm{SCl} A) \leq \operatorname{ISBd} \mathrm{A}$;

(4) $\operatorname{SInt} A \leq A \wedge(\overline{\operatorname{ISBd} A})$.

The following example shows that the equality may not hold in(2), (3) and (4) of Proposition 3.10.

Example 3.11: Choose IFS $M=\left\langle x, \frac{0.5}{\mathrm{a}}+\frac{0.2}{\mathrm{~b}}, \frac{0.5}{\mathrm{a}}+\frac{0.6}{\mathrm{~b}}\right\rangle$ in the IFTS (X $\left.\tau\right)$ defined in example 3.7.

Then, (1) $\operatorname{SCl} \mathrm{M}=1_{\sim}, \operatorname{SCl} \overline{\mathrm{M}}=1_{\sim}, \operatorname{SInt} \mathrm{M}=0_{\sim}, \operatorname{SInt} \overline{\mathrm{M}}=0_{\sim}$

$\operatorname{ISBd} \mathrm{M}=1 \sim \overline{\mathrm{ISBd} M}=1 \sim$.

$\operatorname{ISBd}(\operatorname{SInt} \mathrm{M})=\operatorname{SCl}(\operatorname{SInt} \mathrm{M}) \wedge \mathrm{SCl}(\overline{\operatorname{SInt} \mathrm{M}})=\operatorname{SCl}\left(0_{\sim}\right) \wedge \mathrm{SCl}\left(1_{\sim}\right)=0 \sim$. 
Hence, $\operatorname{ISBd}($ SInt M) $=0 \sim \lesseqgtr \mathrm{ISBd} \mathrm{M}=1 \sim$

(2) $\mathrm{ISBd}(\mathrm{SCl} \mathrm{M})=\mathrm{SCl}(\mathrm{SCl} \mathrm{M}) \wedge \mathrm{SCl}(\overline{\mathrm{SCl} M})=\operatorname{SCl}\left(1_{\sim}\right) \wedge \mathrm{SCl}\left(0_{\sim}\right)=0_{\sim}$.

Hence, ISBd $(\mathrm{SCl} \mathrm{M}) \lesseqgtr \mathrm{ISBd} \mathrm{M}$

(3) $\operatorname{SInt} M=0 \sim \varsubsetneqq \wedge \overline{\operatorname{ISBd} M}=\left\langle x, \frac{0.5}{\mathrm{a}}+\frac{0.2}{\mathrm{~b}}, \frac{0.5}{\mathrm{a}}+\frac{0.6}{\mathrm{~b}}\right\rangle$.

Remark 3.12: In general topology, the following conditions hold.

SBd A $\cap \operatorname{SInt} A=\emptyset$; SInt A U SBd A = SCl A; SInt A U SInt $A^{c} \cup \operatorname{SBd} A=X$.

In intuitionistic fuzzy topology, these may not hold as seen in the following example.

Choose IFS

Example 3.13: Choose IFS $\mathrm{P}=\left\langle x, \frac{0.5}{\mathrm{a}}+\frac{0.6}{\mathrm{~b}}, \frac{0.4}{\mathrm{a}}+\frac{0.3}{\mathrm{~b}}\right\rangle$ in the IFTS ( X $\left.\tau\right)$ defined in example 3.7.

ISBd $\mathrm{P}=\left\langle x, \frac{0.4}{\mathrm{a}}+\frac{0.3}{\mathrm{~b}}, \frac{0.4}{\mathrm{a}}+\frac{0.6}{\mathrm{~b}}\right\rangle, \operatorname{SInt} \mathrm{P}=\left\langle x, \frac{0.4}{\mathrm{a}}+\frac{0.6}{\mathrm{~b}}, \frac{0.4}{\mathrm{a}}+\frac{0.3}{\mathrm{~b}}\right\rangle$

Hence, ISBd P $\wedge$ SInt $\mathrm{P} \neq 0 \sim$.

(2)

SInt P V ISBd P $=\left\langle x, \frac{0.4}{\mathrm{a}}+\frac{0.6}{\mathrm{~b}}, \frac{0.4}{\mathrm{a}}+\frac{0.3}{\mathrm{~b}}\right\rangle \neq 1_{\sim}=\mathrm{SCl} \mathrm{P}$

(3)

SInt $\mathrm{P} \vee \operatorname{SInt} \overline{\mathrm{P}} \vee \operatorname{ISBd} \mathrm{P}=\left\langle x, \frac{0.4}{\mathrm{a}}+\frac{0.6}{\mathrm{~b}}, \frac{0.4}{\mathrm{a}}+\frac{0.3}{\mathrm{~b}}\right\rangle \neq 1_{\sim}$

Theorem 3.14: Let A and B be IFS's in an IFTS( $X \tau)$. Then, $\operatorname{ISBd}(A \vee B) \leq \operatorname{ISBd} A \vee \operatorname{ISBd} B$.

Proof: $\operatorname{ISBd}(\mathrm{A} \vee \mathrm{B})=\operatorname{SCl}(\mathrm{A} \vee \mathrm{B}) \wedge \operatorname{SCl}(\overline{\mathrm{A} \vee \mathrm{B}})$

$$
\begin{aligned}
& \leq(\mathrm{SCl} A \vee \mathrm{SCl} \mathrm{B}) \wedge(\mathrm{SCl} \overline{\mathrm{A}} \wedge \mathrm{SCl} \overline{\mathrm{B}}) \\
& =[\mathrm{SCl} \mathrm{A} \wedge(\mathrm{SCl} \overline{\mathrm{A}} \wedge \mathrm{SCl} \overline{\mathrm{B}})] \vee[\mathrm{SCl} \mathrm{B} \wedge(\mathrm{SCl} \overline{\mathrm{A}} \wedge \mathrm{SCl} \overline{\mathrm{B}})] \\
& =[\mathrm{ISBd} \mathrm{A} \wedge \mathrm{SCl} \overline{\mathrm{B}}] \vee[\mathrm{ISBd} \mathrm{B} \wedge \mathrm{SCl} \overline{\mathrm{A}}] \leq \mathrm{ISBd} \mathrm{A} \vee \mathrm{ISBd} \mathrm{B}
\end{aligned}
$$

Hence, $\operatorname{ISBd}(A \vee B) \leq$ ISBd A $\vee$ ISBd B

In theorem 3.14, the equality may not hold as seen in the following example.

Example 3.15: Choose IFS'S $\mathrm{P}=\left\langle x, \frac{0.5}{\mathrm{a}}+\frac{0.6}{\mathrm{~b}}, \frac{0.4}{\mathrm{a}}+\frac{0.3}{\mathrm{~b}}\right\rangle$

$\mathrm{Q}=\left\langle x, \frac{0.5}{\mathrm{a}}+\frac{0.2}{\mathrm{~b}}, \frac{0.5}{\mathrm{a}}+\frac{0.6}{\mathrm{~b}}\right\rangle$ in the IFTS $(\mathrm{X} \tau)$ defined in example 3.7.

Then $\mathrm{SCl} P=1_{\sim}, \mathrm{SCl} \overline{\mathrm{P}}=\left\langle x, \frac{0.4}{\mathrm{a}}+\frac{0.3}{\mathrm{~b}}, \frac{0.4}{\mathrm{a}}+\frac{0.6}{\mathrm{~b}}\right\rangle$,

ISBd $\mathrm{P}=\left\langle x, \frac{0.4}{\mathrm{a}}+\frac{0.3}{\mathrm{~b}}, \frac{0.4}{\mathrm{a}}+\frac{0.6}{\mathrm{~b}}\right\rangle . \operatorname{SCl} \mathrm{Q}=1_{\sim}, \operatorname{SCl} \overline{\mathrm{Q}}=1_{\sim}$, ISBd $\mathrm{Q}=1_{\sim}$.

$\mathrm{P} \vee \mathrm{Q}=\left\langle x, \frac{0.5}{\mathrm{a}}+\frac{0.6}{\mathrm{~b}}, \frac{0.4}{\mathrm{a}}+\frac{0.3}{\mathrm{~b}}\right\rangle, \mathrm{SCl} \mathrm{P} \vee \mathrm{Q}=1_{\sim}, \mathrm{SCl}(\overline{\mathrm{P} \vee Q})=\left\langle x, \frac{0.4}{\mathrm{a}}+\frac{0.3}{\mathrm{~b}}, \frac{0.4}{\mathrm{a}}+\frac{0.6}{\mathrm{~b}}\right\rangle$

$\operatorname{ISBd}(\mathrm{P} \vee \mathrm{Q})=\left\langle x, \frac{0.4}{\mathrm{a}}+\frac{0.3}{\mathrm{~b}}, \frac{0.4}{\mathrm{a}}+\frac{0.6}{\mathrm{~b}}\right\rangle \neq 1 \sim=\operatorname{ISBd} \mathrm{P} \vee \operatorname{ISBd} \mathrm{Q}$.

Hence, ISBd $(\mathrm{P} \vee \mathrm{Q}) \lesseqgtr$ ISBd P $\vee$ ISBd Q.

Remark 3.16: Let P, Q be IFS's in an IFTS ( $X \tau)$, then the IFS's

ISBd $(P \wedge Q)$ and ISBd $P \wedge$ ISBd $Q$ behave arbitrarily is seen in the following example.

Example 3.17: Choose IFS's $\mathrm{P}=\left\langle x, \frac{0.5}{\mathrm{a}}+\frac{0.6}{\mathrm{~b}}, \frac{0.4}{\mathrm{a}}+\frac{0.3}{\mathrm{~b}}\right\rangle$

$\mathrm{Q}=\left\langle x, \frac{0.5}{\mathrm{a}}+\frac{0.2}{\mathrm{~b}}, \frac{0.5}{\mathrm{a}}+\frac{0.6}{\mathrm{~b}}\right\rangle, \mathrm{R}=\left\langle x, \frac{0.4}{\mathrm{a}}+\frac{0.8}{\mathrm{~b}}, \frac{0.6}{\mathrm{a}}+\frac{0.2}{\mathrm{~b}}\right\rangle$ and 
$\mathrm{S}=\left\langle x, \frac{0.6}{\mathrm{a}}+\frac{0.3}{\mathrm{~b}}, \frac{0.4}{\mathrm{a}}+\frac{0.5}{\mathrm{~b}}\right\rangle$ in the IFTS $(\mathrm{X} \tau)$ of example 3.7.Then,

(i) ISBd $\mathrm{P}=\left\langle x, \frac{0.4}{\mathrm{a}}+\frac{0.3}{\mathrm{~b}}, \frac{0.4}{\mathrm{a}}+\frac{0.6}{\mathrm{~b}}\right\rangle, \operatorname{ISBd} \mathrm{Q}=1 \sim, \operatorname{ISBd}(\mathrm{P} \wedge \mathrm{Q})=1 \sim$

$\operatorname{ISBd} \mathrm{P} \wedge \operatorname{ISBd} \mathrm{Q}=\left\langle x, \frac{0.4}{\mathrm{a}}+\frac{0.3}{\mathrm{~b}}, \frac{0.4}{\mathrm{a}}+\frac{0.6}{\mathrm{~b}}\right\rangle$

Hence, $\operatorname{ISBd}(\mathrm{P} \wedge \mathrm{Q})>\operatorname{ISBd} P \wedge \operatorname{ISBd} Q$.

(ii) ISBd R $=1_{\sim}, \operatorname{ISBd~} \mathrm{S}=\left\langle x, \frac{0.4}{\mathrm{a}}+\frac{0.5}{\mathrm{~b}}, \frac{0.4}{\mathrm{a}}+\frac{0.3}{\mathrm{~b}}\right\rangle$

ISBd R $\wedge$ ISBd $S=\left\langle x, \frac{0.4}{\mathrm{a}}+\frac{0.5}{\mathrm{~b}}, \frac{0.4}{\mathrm{a}}+\frac{0.3}{\mathrm{~b}}\right\rangle$

$\operatorname{ISBd}(\mathrm{R} \wedge \mathrm{S})=\left\langle x, \frac{0.4}{\mathrm{a}}+\frac{0.3}{\mathrm{~b}}, \frac{0.4}{\mathrm{a}}+\frac{0.5}{\mathrm{~b}}\right\rangle$

Hence, ISBd R $\wedge$ ISBd $S>\operatorname{ISBd}(\mathrm{R} \wedge \mathrm{S})$.

Theorem 3.18: For any intuitionistic fuzzy sets $A$ and $B$ in an IFTS $(X \tau)$, $\operatorname{ISBd}(\mathrm{A} \wedge \mathrm{B}) \leq(\mathrm{ISBd} A \wedge \mathrm{SCl} \mathrm{B}) \vee(\operatorname{ISBd} \mathrm{B} \wedge \mathrm{SCl} \mathrm{A})$

Proof:

$$
\begin{aligned}
& \operatorname{ISBd}(A \wedge B)=\operatorname{scl}(A \wedge B) \wedge \operatorname{scl} \overline{(A \wedge B)} \\
& \leq(\operatorname{scl} \mathrm{A} \wedge \mathrm{SCl} \mathrm{B}) \wedge(\operatorname{scl} \overline{\mathrm{A}} \vee \operatorname{scl} \overline{\mathrm{B}}) \\
& =[(\operatorname{scl} \mathrm{A} \wedge \mathrm{scl} \mathrm{B}) \wedge \mathrm{scl} \overline{\mathrm{A}}] \vee[(\operatorname{scl} \mathrm{A} \wedge \mathrm{scl} \mathrm{B}) \wedge \mathrm{scl} \overline{\mathrm{B}}] \\
& =[(\operatorname{scl} \mathrm{A} \wedge \operatorname{scl} \overline{\mathrm{A}}) \wedge \mathrm{scl} \mathrm{B}] \vee[(\operatorname{scl} \mathrm{B} \wedge \mathrm{scl} \overline{\mathrm{B}}) \wedge \mathrm{sCl} \mathrm{A}]
\end{aligned}
$$

Hence, $\operatorname{ISBd}(\mathrm{A} \wedge \mathrm{B}) \leq($ ISBd $\mathrm{A} \wedge \mathrm{SCl} \mathrm{B}) \vee($ ISBd B $\wedge \mathrm{SCl} \mathrm{A})$.

Corollary 3.19: For any intuitionistic fuzzy sets A and B in an IFTS ( X $\tau$ ), then ISBd $(A \wedge B) \leq \operatorname{ISBd} A \vee \operatorname{ISBd} B$.

The equality in the above theorem may not hold as seen in the following example.

Example 3.20: Choose IFS's $\mathrm{R}=\left\langle x, \frac{0.4}{\mathrm{a}}+\frac{0.8}{\mathrm{~b}}, \frac{0.6}{\mathrm{a}}+\frac{0.2}{\mathrm{~b}}\right\rangle$ and $\mathrm{S}=\left\langle x, \frac{0.6}{\mathrm{a}}+\frac{0.3}{\mathrm{~b}}, \frac{0.4}{\mathrm{a}}+\frac{0.5}{\mathrm{~b}}\right\rangle$ in the IFTS $(\mathrm{X} \tau)$ of example 3.7.

Then, ISBd $\mathrm{R}=1_{\sim}$, ISBd $\mathrm{S}=\left\langle x, \frac{0.4}{\mathrm{a}}+\frac{0.5}{\mathrm{~b}}, \frac{0.4}{\mathrm{a}}+\frac{0.3}{\mathrm{~b}}\right\rangle$

$($ ISBd R $\wedge \mathrm{SCl}$ S $) \vee($ ISBd S $\wedge \mathrm{SCl} \mathrm{R})=1_{\sim} \vee\left\langle x, \frac{0.4}{\mathrm{a}}+\frac{0.5}{\mathrm{~b}}, \frac{0.4}{\mathrm{a}}+\frac{0.3}{\mathrm{~b}}\right\rangle=1_{\sim}$.

$\operatorname{ISBd}(\mathrm{R} \wedge \mathrm{S})=\left\langle x, \frac{0.4}{\mathrm{a}}+\frac{0.3}{\mathrm{~b}}, \frac{0.4}{\mathrm{a}}+\frac{0.5}{\mathrm{~b}}\right\rangle$

Hence, ISBd $(\mathrm{R} \wedge \mathrm{S}) \lesseqgtr($ ISBd $\mathrm{R} \wedge \mathrm{SCl} \mathrm{S}) \vee($ ISBd $\mathrm{S} \wedge \mathrm{SCl} \mathrm{R})$.

Proposition 3.21: For any IFS A in an IFTS X, then,

(1) ISBd $($ ISBd A) $\leq$ ISBd A ,

(2) $\operatorname{ISBd}(\operatorname{ISBd}(\operatorname{ISBd} A)) \leq \operatorname{ISBd}($ ISBd A) 


\section{Proof:}

(1) $\operatorname{ISBd}(\operatorname{ISBd} A)=\operatorname{SCl}(\operatorname{ISBd} A) \wedge \operatorname{SCl}(\overline{\operatorname{ISBd} A})$

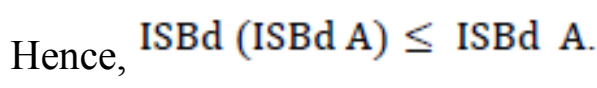

$$
\leq \operatorname{SCl}(\text { ISBd A }) \leq \operatorname{ISBd~A} \text { of proposition } 3.8
$$

(2) $\operatorname{ISBd}(\operatorname{ISBd}(\operatorname{ISBd} A))=\operatorname{SCl}(\operatorname{ISBd}(\operatorname{ISBd} A)) \wedge \operatorname{SCl}(\overline{\text { ISBd }(\operatorname{ISBd} \mathrm{A})})$

$$
\leq \operatorname{ISBd}(\operatorname{ISBd} A) \wedge \operatorname{SCl}(\overline{\text { ISBd }(\operatorname{ISBd} A)}) \leq \operatorname{ISBd}(\operatorname{ISBd} A)
$$

Hence, $\operatorname{ISBd}(\operatorname{ISBd}(\operatorname{ISBd} A)) \leq \operatorname{ISBd}(\operatorname{ISBd} A)$.

Remark 3.22: The equality may not hold in proposition 3.21 (1) as seen in the following example. We could not find an example to show that equality in proposition 3.21 (2) may not hold.

Example 3.23: Choose IFS $\mathrm{M}=\left\langle x, \frac{0.5}{\mathrm{a}}+\frac{0.2}{\mathrm{~b}}, \frac{0.5}{\mathrm{a}}+\frac{0.6}{\mathrm{~b}}\right\rangle$ in the IFTS (X $\tau$ ) defined in example 3.7.

Then, (1) $\operatorname{scl} \mathrm{M}=1_{\sim}, \operatorname{scl} \overline{\mathrm{M}}=1_{\sim}$, ISBd $\mathrm{M}=1_{\sim}, \overline{\mathrm{ISBd} M}=0_{\sim}$

$\operatorname{ISBd}(\operatorname{ISBd} \mathrm{M})=\operatorname{scl}(\operatorname{ISBd} \mathrm{M}) \wedge \operatorname{SCl}(\overline{\mathrm{ISBd} M})=\operatorname{scl} 1_{\sim} \wedge \operatorname{SCl} 0_{\sim}=1 \sim \wedge 0_{\sim}=0_{\sim}$

Hence, ISBd (ISBd M) $\neq$ ISBd M.

\section{Intuitionistic Fuzzy product related spaces}

K.K.Azad[3] introduced the concept of fuzzy product related spaces. H.M.Hanafy[7] extend this concept to intuitionistic fuzzy topological spaces as follows:

Definition 4.1: [7] If $A=\left\langle x, \mu_{\mathrm{A}}(x), \gamma_{\mathrm{A}}(x)\right\rangle$ and $B=\left\langle y, \mu_{\mathrm{B}}(y), \gamma_{\mathrm{B}}(y)\right\rangle$ be IFSs of $\mathrm{X}$ and $\mathrm{Y}$ respectively. Then the product of intuitionistic fuzzy sets $\mathrm{A}$ and $\mathrm{B}$ be defined by,

$A \times B=\left\langle(x, y), \mu_{\mathrm{A} \times \mathrm{B}}(x, y), \gamma_{\mathrm{A} \times \mathrm{B}}(x, y)\right\rangle$, is $\mathrm{FSS}$ in $\mathrm{X} \times \mathrm{Y}$;

where $\mu_{(\mathbf{A} \times \mathbf{B})}(x, y)=\min \left\{\mu_{\mathbf{A}}(x), \mu_{\mathbf{B}}(y)\right\}$ for each $(x, y) \in \mathrm{X} \times \mathrm{Y}$;

$$
\gamma_{(\mathbf{A} \times \mathbf{B})}(x, y)=\max \left\{\gamma_{\mathbf{A}}(x), \gamma_{\mathbf{B}}(y)\right\} \text { for each }(x, y) \in \mathrm{X} \times \mathrm{Y}
$$

and $0 \leq \mu_{\mathrm{A} \times \mathrm{B}}(x, y)+\gamma_{\mathrm{A} \times \mathrm{B}}(x, y) \leq 1$. It may be notice that $\overline{A \times B}=\left\langle(x, y), \gamma_{\mathrm{A} \times \mathrm{B}}(x, y), \mu_{\mathrm{A} \times \mathrm{B}}(x, y)\right\rangle$. The following lemma is needed in sequel.

Lemma 4.2: Let A,B,C and D be a IFS in IFTS $X$, then $(A \wedge B) \times(C \wedge D)=(A \times D) \wedge(B \times C)$ 


$$
\begin{aligned}
& \text { Proof: } \mu_{(\mathrm{A} \wedge \mathrm{B}) \times(\mathrm{C} \wedge \mathrm{D})}(x, y)=\min \left\{\mu_{(\mathrm{A} \wedge \mathrm{B})}(x), \mu_{(\mathrm{C} \wedge \mathrm{D})}(y)\right\} \\
& =\min \left\{\min \left\{\mu_{\mathrm{A}}(x), \mu_{\mathrm{B}}(x)\right\}, \min \left\{\mu_{\mathrm{C}}(y), \mu_{\mathrm{D}}(y)\right\}\right] \\
& =\min \left\{\min \left\{\mu_{\mathrm{A}}(x), \mu_{\mathrm{D}}(y)\right\}, \min \left\{\mu_{\mathrm{B}}(x), \mu_{\mathrm{C}}(y)\right\}\right\} \\
& =\min \left\{\mu_{(\mathbf{A} \times \mathbf{D})}(x, y), \mu_{(\mathbf{B} \times \mathbf{C})}(x, y)\right\} \\
& \mu_{(\mathrm{A} \wedge \mathrm{B}) \times(\mathrm{C} \wedge \mathrm{D})}(x, y)=\mu_{(\mathrm{A} \times \mathrm{D}) \wedge(\mathrm{B} \times \mathrm{C})}(x, y) \\
& \gamma_{(\mathrm{A} \wedge \mathrm{B}) \times(\mathrm{C} \wedge \mathrm{D})}(x, y)=\max \left\{\gamma_{(\mathrm{A} \wedge \mathrm{B})}(x), \gamma_{(\mathrm{C} \wedge \mathrm{D})}(y)\right\} \\
& =\max \left\{\max \left\{\gamma_{A}(x), \gamma_{B}(x)\right\}, \max \left\{\gamma_{C}(y), \gamma_{D}(y)\right\}\right\} \\
& =\max \left\{\max \left\{\gamma_{A}(x), \gamma_{D}(y)\right\}, \max \left\{\gamma_{B}(x), \gamma_{C}(y)\right\}\right\} \\
& =\max \left\{\gamma_{(\mathrm{A} \times \mathrm{D})}(x, y), \gamma_{(\mathrm{B} \times \mathrm{C})}(x, y)\right\}
\end{aligned}
$$

$\gamma_{(\mathrm{A} \wedge \mathrm{B}) \times(\mathrm{C} \wedge \mathrm{D})}(x, y)=\gamma_{(\mathrm{A} \times \mathrm{D}) \wedge(\mathrm{B} \times \mathrm{C})}(x, y)$.

Hence, $(A \wedge B) x(C \wedge D)=(A x d) \wedge(B x C)$

Definition 4.3: [7] An IFTS $\left(\mathrm{X}, \tau_{\mathrm{X}}\right)$ is product related to another IFTS $\left(\mathrm{Y}, \tau_{\mathrm{Y}}\right)$ if for any intuitionistic fuzzy sets $U$ of $X$ and $V$ of $Y$, whenever $\bar{A} \notin U$ and $\bar{B} \notin V$ imply $\bar{A} x$ $1_{\sim} \vee 1_{\sim} \times \bar{B} \geq U \times V$, where $A \in \tau_{X}$ and $B \in \tau_{Y}$ there exist $A_{1} \in \tau_{X}$ and $B_{1} \in \tau_{Y}$ such that $\overline{A_{1}} \geq U$ or $\overline{B_{1}} \geq V$ and $\overline{A_{1}} \times 1_{\sim} \vee 1_{\sim} \times \overline{B_{1}}=\bar{A} \times 1_{\sim} \vee 1_{\sim} \times \bar{B}$.

Lemma 4.4: [7] If $A$ is an IFS of $X$ and $B$ is an IFS of $Y$, then

(1) $\left(\mathrm{A} \times 1_{\sim}\right) \cap\left(1_{\sim} \times \mathrm{B}\right)=\mathrm{A} \times \mathrm{B}$;

(2) $\overline{\mathrm{A} \times \mathrm{B}}=\left(\overline{\mathrm{A}} \times 1_{\sim}\right) \cup\left(1_{\sim} \times \overline{\mathrm{B}}\right)$;

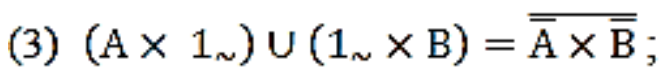

Proof: Let $=\left\langle x, \mu_{\mathrm{A}}(x), \gamma_{\mathrm{A}}(x)\right\rangle, B=\left\langle y, \mu_{\mathrm{B}}(y), \gamma_{\mathrm{B}}(y)\right\rangle$.

(1) Since $\mathrm{A} \times 1_{\sim}=\left\langle x, \min \left(\mu_{\mathrm{A}}, 1_{\sim}\right), \max \left(\gamma_{\mathrm{A}}, 0_{\sim}\right)\right\rangle=\left\langle x, \mu_{\mathrm{A}}(x), \gamma_{\mathrm{A}}(x)\right\rangle=\mathrm{A}$.

Similarly $1_{\sim} \times B=\left\langle y, \min \left(1_{\sim}, \mu_{B}\right), \max \left(0_{\sim}, \gamma_{B}\right)\right\rangle=\left\langle y, \mu_{B}(x), \gamma_{B}(x)\right\rangle=B$.

We have

$\left(\mathrm{A} \times 1_{\sim}\right) \cap\left(1_{\sim} \times \mathrm{B}\right)=\mathrm{A}(\mathrm{x}) \cap \mathrm{B}(\mathrm{y})=\left\langle(x, y), \mu_{\mathrm{A}}(x) \wedge \mu_{\mathrm{B}}(y), \gamma_{\mathrm{A}}(x) \vee \gamma_{\mathrm{B}}(y)\right\rangle$

Hence, $\left(\mathrm{A} \times 1_{\sim}\right) \cap\left(1_{\sim} \times \mathrm{B}\right)=A \times B$.

(2) Since $\bar{A}=\left\langle x, \gamma_{\mathrm{A}}(x), \mu_{\mathrm{A}}(x)\right\rangle, \bar{B}=\left\langle y, \gamma_{\mathrm{B}}(y), \mu_{\mathrm{B}}(y)\right\rangle$

$\overline{\mathrm{A}} \times 1_{\sim}=\left\langle(x, y), \min \left(\gamma_{\mathrm{A}}(\mathrm{x}), 1_{\sim}(\mathrm{y})\right), \max \left(\mu_{\mathrm{A}}(\mathrm{x}), 0_{\sim}(\mathrm{y})\right)\right\rangle=\left\langle(x, y), \gamma_{\mathrm{A}}(x), \mu_{\mathrm{A}}(x)\right\rangle$

$1_{\sim} \times \overline{\mathrm{B}}=\left\langle(x, y), \min \left(1_{\sim}(\mathrm{x}), \mu_{\mathrm{B}}(\mathrm{y})\right), \max \left(0_{\sim}(\mathrm{x}), \gamma_{\mathrm{B}}(\mathrm{y})\right)\right\rangle=\left\langle(x, y), \gamma_{\mathrm{B}}(y), \mu_{\mathrm{B}}(y)\right\rangle$

$\left(\overline{\mathrm{A}} \times 1_{\sim}\right) \cup\left(1_{\sim} \times \overline{\mathrm{B}}\right)=\left\langle(x, y), \max \left(\gamma_{\mathrm{A}}(x), \gamma_{\mathrm{B}}(\mathrm{y})\right), \min \left(\mu_{\mathrm{A}}(x), \mu_{\mathrm{B}}(\mathrm{y})\right)\right\rangle=\overline{\mathrm{A} \times \mathrm{B}}$

(3) Follows from (1) and (2). 
Lemma 4.5: Let $A$ and $B$ be IFSCS's in IFTS's $X$ and $Y$ respectively. Then $A \times B$ is an IFSCS in the intuitionistic fuzzy product topological space (IFPTS) of $\mathrm{X} \times \mathrm{Y}$.

Proof: Let $A=\left\langle x, \mu_{A}(x), \gamma_{A}(x)\right\rangle, B=\left\langle y, \mu_{B}(y), \gamma_{B}(y)\right\rangle$. From Lemma 4.4 we have

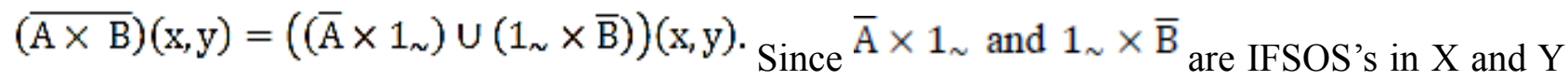
respectively, hence $\left(\overline{\mathrm{A}} \times 1_{\sim}\right) \cup\left(1_{\sim} \times \overline{\mathrm{B}}\right)$ is IFSOS of $\mathrm{X} \times \mathrm{Y}$. Hence $\overline{\mathrm{A} \times \mathrm{B}}$ is an IFSOS of $\mathrm{X} \times \mathrm{Y}$ and consequently $\mathrm{A} \times \mathrm{B}$ is an IFSCS of $\mathrm{X} \times \mathrm{Y}$.

Theorem 4.6: If $A$ and $B$ are IFS's of IFTS's $X$ and $Y$ respectively, then

(1) $\mathrm{SCl} \mathrm{A} \times \mathrm{SCl} \mathrm{B} \geq \mathrm{SCl}(\mathrm{A} \times \mathrm{B})$;

(2) $\operatorname{SInt} A \times \operatorname{SInt} B \leq \operatorname{SInt}(A \times B)$.

Proof: (1) Since $A \leq S C l(A)$ and $B \leq S C l(B)$, hence $A \times B \leq \operatorname{SCl}(A) \times \operatorname{SCl}(B)$ implies $\operatorname{SCl}(A \times B) \leq \operatorname{SCl}(\operatorname{SCl}(A) \times \operatorname{SCl}(B)) \quad$ and $\quad$ from $\quad$ Lemma 4.5 we have $\operatorname{scl}(A \times B) \leq \operatorname{SCl}(A) \times \operatorname{SCl}(B)$.

(2) Follows from (1) and using the facts that $\overline{\operatorname{SCl}(\mathrm{C})}=\operatorname{SInt}(\overline{\mathrm{C}})$ and $\overline{\operatorname{SInt}(\mathrm{C})}=\operatorname{SCl}(\overline{\mathrm{C}})$.

Theorem 4.7: Let $(\mathrm{X}, \tau)$ and $(\mathrm{Y}, \delta)$ be product related IFTSs. Then for a IFS A of X and a IFS B of Y, then

(1) $\operatorname{SCl}(\mathrm{A} \times \mathrm{B})=\mathrm{SCl} \mathrm{A} \times \mathrm{SCl}$;

(2) $\operatorname{SInt}(A \times B)=\operatorname{SInt} A \times \operatorname{SInt} B$.

Proof: (1) For intuitionistic fuzzy sets $A_{i}{ }^{\prime}$ of $X$ and ${ }^{B_{j}}{ }^{\prime}$ of $Y$ then note that

(i) $\inf \left\{A_{i}, B_{j}\right\}=\min \left(\inf A_{i}, \inf B_{j}\right)$;

(ii) $\inf \left\{A_{\mathbf{i}} \times 1_{\sim}\right\}=\left(\operatorname{inf~} A_{i}\right) \times 1_{\sim}$;

(iii) $\inf \left\{1_{\sim} \times B_{j}\right\}=1_{\sim} \times\left(\inf B_{j}\right)$

By theorem4.6, sufficient to show that $\mathrm{SCl}(\mathrm{A} \times \mathrm{B}) \geq \mathrm{SCl} \mathrm{A} \times \mathrm{SCl} \mathrm{B}$.

Let $A_{i} \in \operatorname{IFSO}(X)$ and $B_{j} \in \operatorname{IFSO}(Y)$. Then,

$$
\begin{aligned}
\operatorname{scl}(A \times B) & =\inf \left\{\left(\overline{A_{1} \times B_{j}}\right):\left(\overline{A_{1} \times B_{j}}\right) \geq A \times B\right\} \\
& =\inf \left\{\overline{A_{1}} \times 1_{\sim} \vee 1_{\sim} \times \overline{B_{j}}: \overline{A_{1}} \times 1_{\sim} \vee 1_{\sim} \times B_{j} \geq A \times B\right\} \\
& =\inf \left\{\overline{A_{1}} \times 1_{\sim} \vee 1_{\sim} \times \overline{B_{j}}: \overline{A_{1}} \geq A(o r) \overline{B_{j}} \geq B\right\} \\
& =\min \left(\inf \left\{\overline{A_{1}} \times 1_{\sim} \vee 1_{\sim} \times \overline{B_{j}}: \overline{A_{1}} \geq A\right\}, \inf \left\{\overline{A_{1}} \times 1_{\sim} \vee 1_{\sim} \times \overline{B_{j}}: \overline{B_{j}} \geq B\right\}\right)
\end{aligned}
$$


Since $\inf \left\{\overline{\mathrm{A}_{1}} \times 1_{\sim} \vee 1_{\sim} \times \overline{B_{\mathrm{j}}}: \overline{\mathrm{A}_{1}} \geq A\right\} \geq \inf \left\{\overline{\mathrm{A}_{1}} \times 1_{\sim}: \overline{\mathrm{A}_{1}} \geq A\right\}$

$$
=\inf \left\{\overline{\mathrm{A}_{1}}: \overline{\mathrm{A}_{1}} \geq \mathrm{A}\right\} \times 1_{\sim}=(\mathrm{SCl} \mathrm{A}) \times 1_{\sim}
$$

$$
\begin{aligned}
\inf \left\{\overline{\mathrm{A}_{1}} \times 1_{\sim} \vee 1_{\sim} \times \overline{\mathrm{B}}_{\mathrm{j}}: \overline{\mathrm{B}_{\mathrm{j}}} \geq \mathrm{B}\right\} & \geq \inf \left\{\overline{\mathrm{B}_{\mathrm{j}}} \times 1_{\sim}: \overline{\mathrm{B}_{\mathrm{j}}} \geq \mathrm{B}\right\} \\
& =1_{\sim} \times \inf \left\{\overline{\mathrm{B}}_{\mathrm{j}}: \overline{\mathrm{B}}_{\mathrm{j}} \geq \mathrm{B}\right\}=1_{\sim} \times(\operatorname{scl} \mathrm{B})
\end{aligned}
$$

Hence, $\operatorname{SCl}(\mathrm{A} \times \mathrm{B}) \geq \min \left((\operatorname{SCl} \mathrm{A}) \times 1_{\sim}, 1_{\sim} \times(\mathrm{SCl} \mathrm{B})\right)=\operatorname{scl} \mathrm{A} \times \mathrm{SCl} \mathrm{B}$.

(2) This follows from (1) and using the facts that $\overline{\operatorname{SCl}(\mathrm{C})}=\operatorname{SInt}(\overline{\mathrm{C}})$ and $\overline{\operatorname{SInt}(\mathrm{C})}=\operatorname{SCl}(\overline{\mathrm{C}})$.

Theorem 4.8: Let Let $X_{i}, i=1,2,3, \ldots \ldots . n$, be a family of product related intuitionistic fuzzy topological spaces. If each $A_{i}$ is a IFS in $X_{i}$, then

$\operatorname{ISBd} \prod_{i=1}^{\mathrm{n}} \mathrm{A}_{\mathrm{i}}=\left[\operatorname{ISBd} \mathrm{A}_{1} \times \operatorname{SCl} \mathrm{A}_{2} \times \ldots \ldots \ldots . . . . \mathrm{SC} l \mathrm{~A}_{\mathrm{n}}\right]$

$$
\begin{aligned}
& \vee\left[\operatorname{SCl} \mathrm{A}_{1} \times \operatorname{ISBd~} \mathrm{A}_{2} \times \operatorname{SCl} \mathrm{A}_{3} \ldots \ldots \ldots . . \times \mathrm{SCl} \mathrm{A}_{\mathrm{n}}\right] \\
& \vee \ldots \ldots \ldots \ldots \vee\left[S C l A_{1} \times \operatorname{SCl} A_{2} \times \ldots \ldots \ldots . . \ldots \text { ISBd } A_{n}\right]
\end{aligned}
$$

Proof: It suffices to prove this for $n=2$.

$$
\begin{aligned}
& \operatorname{ISBd}\left(A_{1} \times A_{2}\right)=\operatorname{SCl}\left(A_{1} \times A_{2}\right) \wedge \operatorname{SCl}\left(\overline{A_{1} \times A_{2}}\right) \\
& =\operatorname{SCl}\left(A_{1} \times A_{2}\right) \wedge\left(\operatorname{SInt}\left(A_{1} \times A_{2}\right)\right) \\
& =\left(\operatorname{SCl} A_{1} \times \operatorname{SCl} A_{2}\right) \wedge\left(\overline{\operatorname{SInt} A_{1} \times \operatorname{SInt} A_{2}}\right) \\
& =\left(\operatorname{SCl} \mathrm{A}_{1} \times \operatorname{SCl} \mathrm{A}_{2}\right) \wedge\left[\overline{\left(\operatorname{SInt} \mathrm{A}_{1} \wedge \mathrm{SCl} \mathrm{A}_{1}\right) \times\left(\operatorname{SInt} \mathrm{A}_{2} \wedge \mathrm{SCl} \mathrm{A}_{2}\right)}\right] \\
& =\left(\operatorname{SCl} A_{1} \times \operatorname{SCl~A} 2\right) \wedge\left[\left(\overline{\operatorname{SInt} A_{1} \wedge \operatorname{SCl} A_{1}}\right) \times 1 \vee 1 \times\left(\overline{\operatorname{SInt} A_{2} \wedge \operatorname{SCl} A_{2}}\right)\right] \\
& \text { by Lemma } 4.4 \text { of (2) } \\
& =\left(\operatorname{SCl} \mathrm{A}_{1} \times \operatorname{SCl} \mathrm{A}_{2}\right) \wedge\left[\left(\operatorname{SCl} \overline{\mathrm{A}_{1}} \vee \operatorname{Sint} \overline{\mathrm{A}_{1}}\right) \times 1 \vee 1 \times\left(\operatorname{SCl} \overline{\mathrm{A}_{2}} \vee \operatorname{Sint} \overline{\mathrm{A}_{2}}\right)\right] \\
& =\left(\operatorname{SCl} \mathrm{A}_{1} \times \mathrm{SCl} \mathrm{A}_{2}\right) \wedge\left[\left(\mathrm{SCl} \overline{\mathrm{A}_{1}} \times 1\right) \vee\left(1 \times \mathrm{SCl} \overline{\mathrm{A}_{2}}\right)\right] \quad \text { by Lemma } 4.2 \\
& =\left[\left(\operatorname{SCl} \mathrm{A}_{1} \times \mathrm{SCl} \mathrm{A}_{2}\right) \wedge\left(\mathrm{SCl} \overline{\mathrm{A}_{1}} \times 1\right)\right] \vee\left[\left(\operatorname{SCl} \mathrm{A}_{1} \times \mathrm{SCl} \mathrm{A}_{2}\right) \wedge\left(1 \times \mathrm{SCl} \overline{\mathrm{A}_{2}}\right)\right] \\
& =\left[\left(\operatorname{SCl} \mathrm{A}_{1} \wedge \mathrm{SCl} \overline{\mathrm{A}_{1}}\right) \times\left(1 \wedge \mathrm{SCl} \mathrm{A}_{2}\right)\right] \vee\left[\left(\operatorname{SCl} \mathrm{A}_{1} \wedge 1\right) \times\left(\operatorname{scl} \mathrm{A}_{2} \wedge \mathrm{SCl} \overline{\mathrm{A}_{2}}\right)\right]
\end{aligned}
$$

Hence, $\operatorname{ISBd}\left(\mathrm{A}_{1} \times \mathrm{A}_{2}\right)=\left(\operatorname{ISBd~A_{1}\times \operatorname {SCl~A}}\right) \vee\left(\mathrm{SCl} \mathrm{A} \times \operatorname{ISBd~A_{2}}\right)$.

\section{Intuitionistic Fuzzy continuous function and Irresolute function:}

Definition 5.1: [6] Let $(X, \tau)$ and $(Y, \delta)$ be two IFTS's. A function $f:(X, \tau) \rightarrow(Y, \delta)$ is said to be intuitionistic fuzzy semi continuous, if the pre image of each IFOS in Y is IFSOS in $\mathrm{X}$.

Theorem 5.2:[11] Let $(X, \tau)$ and $(Y, \delta)$ be two IFTS's and let $f: X \rightarrow Y$ be a mapping. Then, the following conditions are equivalent 
(1) $f$ is intuitionistic fuzzy semi continuous,

(2) $f^{1}(\mathrm{~B})$ is an IFSCS in X for each IFCS B in Y,

(3) $\left(\mathrm{SCl}\left(f^{l} \mathrm{~B}\right)\right) \leq\left(f^{l}(\mathrm{~B})\right)$, for each intuitionistic fuzzy set $\mathrm{B}$ in $\mathrm{Y}$,

(4) $f^{l}(\operatorname{Int}(\mathrm{B})) \leq \operatorname{SInt}\left(f^{l}(\mathrm{~B})\right)$, for each intuitionistic fuzzy set B in $\mathrm{Y}$,

(5) $f^{l}(\mathrm{~B}) \leq \mathrm{Cl}$. (SInt $\left(f^{l}(\mathrm{~B})\right)$, for each intuitionistic fuzzy set B in $\mathrm{Y}$.

Theorem 5.3: Let $X$ be a IFTS and $f: X \rightarrow Y$ be a intuitionistic fuzzy semi continuous function. Then, $\operatorname{ISBd} f^{l}(\mathrm{~B}) \leq(\operatorname{IBd} \mathrm{B})$, for any IFS B in Y.

Proof: Let be intuitionistic fuzzy semi continuous and B be a IFS in Y. Then, $C l B$ is intuitionistic fuzzy closed in Y. Which implies that $f^{l}(C l B)$ is intuitionistic fuzzy semi closed in $\mathrm{X}$.

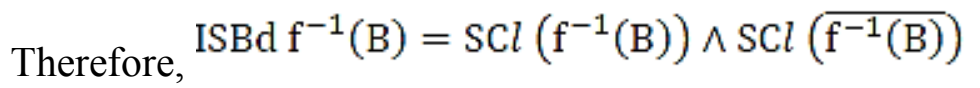

$$
\begin{aligned}
& \leq \mathrm{f}^{-1}(\mathrm{Cl} \mathrm{B}) \wedge \mathrm{SCl} \mathrm{f}^{-1}(\overline{\mathrm{B}}) \leq \mathrm{f}^{-1}(\mathrm{ClB}) \wedge \mathrm{f}^{-1}(\mathrm{Cl} \overline{\mathrm{B}}) \\
& \leq \mathrm{f}^{-1}(\mathrm{Cl} \mathrm{B} \wedge \mathrm{Cl} \overline{\mathrm{B}})=\mathrm{f}^{-1}(\mathrm{IBd} \mathrm{B})
\end{aligned}
$$

Hence, $\operatorname{ISBd} \mathrm{f}^{-1}(\mathrm{~B}) \leq \mathrm{f}^{-1}($ IBd $B)$.

Definition 5.4: $[11]$ Let $(\mathrm{X}, \tau)$ and $(\mathrm{Y}, \delta)$ be two IFTS's. A function $\mathrm{f:}(\mathrm{X}, \tau) \rightarrow(\mathrm{Y}, \delta)$ is said to be intuitionistic fuzzy irresolute if and only if the pre image of each IFSOS in $\mathrm{Y}$ is and IFSOS in $\mathrm{X}$.

Theorem 5.5: [11] Let $(X, \tau)$ and $(Y, \delta)$ be two IFTS's and let $f: X \rightarrow Y$ be a mapping. Then, the following conditions are equivalent,

(1) $f$ is intuitionistic fuzzy irresolute,

(2) $f^{1}(\mathrm{~B})$ is an IFSCS in X for each IFSCS B in Y,

(3) $f^{1}(\mathrm{SCl} \mathrm{A}) \leq \mathrm{SCl}\left(f^{1}(\mathrm{~A})\right)$ for each intuitionistic fuzzy set $\mathrm{A}$ in $\mathrm{X}$,

(4) $\mathrm{SCl}\left(f^{l}(\mathrm{~B})\right) \leq f^{l}$ ( $\left.\mathrm{SCl} \mathrm{B}\right)$ for each intuitionistic fuzzy set $\mathrm{B}$ in $\mathrm{Y}$,

$(5) f^{l}(\mathrm{SIntB}) \leq \operatorname{Int}\left(f^{l}(\mathrm{~B})\right)$ for each intuitionistic fuzzy set $\mathrm{B}$ in $\mathrm{Y}$.

The following theorem gives a necessary condition of intuitionistic fuzzy irresolute functions in terms of intuitionistic fuzzy semi boundary.

Theorem 5.6: Let $\mathrm{f}: \mathrm{X} \rightarrow \mathrm{Y}$ be a intuitionistic fuzzy irresolute function.

Then, ISBd $\mathrm{f}^{-1}(\mathrm{~B}) \leq \mathrm{f}^{-1}$ (ISBd B) for any IFS B in $\mathrm{Y}$.

Proof: Let B be a IFS in Y. Then, $S C l$ is intuitionistic fuzzy closed in Y. Which implies that $f^{1}$ (SCl $\mathrm{B})$ is intuitionistic fuzzy semi closed in $\mathrm{X}$.

Therefore, ${ }^{I S B d ~ f^{-1}(B)}=\operatorname{SCl}\left(\mathrm{f}^{-1}(\mathrm{~B})\right) \wedge \mathrm{SCl}\left(\overline{\mathrm{f}^{-1}(\mathrm{~B})}\right)$

$$
\begin{aligned}
& \leq \mathrm{f}^{-1}(\operatorname{scl}(\mathrm{B})) \wedge \mathrm{SCl}\left(\mathrm{f}^{-1}(\overline{\mathrm{B}})\right) \leq \mathrm{f}^{-1}(\operatorname{scl}(\mathrm{B})) \wedge \mathrm{f}^{-1}(\operatorname{scl}(\overline{\mathrm{B}})) \\
& \leq \mathrm{f}^{-1}(\operatorname{scl} \mathrm{B} \wedge \mathrm{SCl} \overline{\mathrm{B}})=\mathrm{f}^{-1}(\mathrm{ISBd} \mathrm{B})
\end{aligned}
$$

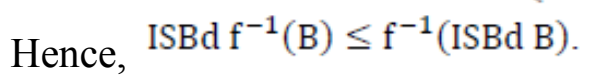

Acknowledgement: The authors are highly grateful to referees for valuable comments and suggestions for improving the paper. 


\section{REFERENCES}

[1] K. T.Atanassov, Intuitionistic fuzzy sets, Fuzzy sets and system 20 (1986), no.1, 87-96.

[2] M.Athar and B.Ahmad, Fuzzy boundary and Fuzzy semi boundary, Advances inFuzzy systems,Article ID.586893 (2008) 1-9 pages.

[3] K.K.Azad, On fuzzy continuity, fuzzy almost continuity and fuzzy weakly continuity, J. Math. Anal. Appl. 82(1981),14-32

[4] C.L.Chang, Fuzzy topological spaces,J. Math. Anal. Appl. 24(1968), no.1, 182-190.

[5] D.Coker, An introduction to intuitionistic fuzzy topological spaces, Fuzzy setsand system 88 (1997),no.1, 81-89.

[6] H.G r̈cay, D.Coker and A.H.Es, On fuzzy continuity in intuitionistic fuzzytopological spaces, J. Fuzzy Math.5(1997), no.2, 365-378.

[7] I.M. Hanafy, completely continuous functions in intuitionistic fuzzy topological spaces, Czechoslovak Math. J. 53(4) (2003), 793-803.

[8] P. M. Pu and Y. M. Liu, Fuzzy topology I-Neighborhood structure of a fuzzy point and Mooresmith convergence, J. Math. Anal. Appl., Vol.76, No.2(1980), 571-599.

[9] V.Thiripurasundari and S.Murugesan, Intuitionistic fuzzy boundary and intuitionistic fuzzy continuous functions, (accepted in "The Journal of the Indian Academy of Mathematics").

[10] L.A.Zadeh, Fuzzysets, Information and control 8(1965),338-353.

[11] Zhen-Guoxu and Fu-Gui Shi, Some Weakly Mappings on Intuitionistic Fuzzy Topological Spaces, TAMKANG Journal of Mathematics, Vol39, No 1,25-32, Spring 2008. 